\title{
ENSEÑANZA DE LA FILOSOFÍA: ¿QUÉ SE LE ESCAPA AL ENFOQUE ACADÉMICO?
}

\section{Joaquín Darío Huertas Ruiz*}

\begin{abstract}
RESUMEN: El presente trabajo describe lo que el autor denomina el enfoque académico de la enseñanza de la filosofía. La aclaración de tal afirmación implica reconocer las directrices que este esquema impone a la enseñanza de la Filosofía y los cuestionamientos generados respecto a las consecuencias de esta perspectiva en los cursos de la educación secundaria, la formación profesional de los profesores de Filosofía y la enseñanza misma.
\end{abstract}

Palabras clave: enseñanza de la filosofía; enfoque académico; didáctica; educación media; profesores; filosofar.

RESUMO: O presente trabalho descreve o que o autor denomina o enfoque acadêmico do ensino da filosofia. Esclarecer esta afirmação implica reconhecer as diretrizes que este esquema exige ao ensino da Filosofia e os questionamentos gerados sobre as consequências desta perspectiva nos cursos de ensino médio, a formação profissional dos professores de Filosofia e próprio ensino.

Palavras-chave: ensino da filosofia; enfoque acadêmico; didática; ensino médio; professores; filosofar.

\section{Introducción}

Existe gran preocupación en el medio académico por la educación secundaria y en nuestro caso específico, por el lugar de la Filosofía en ella, tanto en la preparación con miras a la educación superior, como para la formación del individuo. Es común escuchar a los filósofos profesionales quejarse del casi nulo interés de las mayorías por el cultivo de la disciplina, atribuyéndosele a los docentes de bachillerato, los contenidos y metodologías aplicadas por estos y al desinterés generalizado por las humanidades y demás formas de conocimiento. En el mismo sentido, muchos egresados de la

\footnotetext{
* Licenciado en Filosofía e Historia y Magister en Filosofía Latinoamericana por la Universidad de Santo Tomas; Especialista en Teorías, Técnicas y Métodos de Investigación Social, de la Universidad Pedagógica Nacional y actualmente candidato a Doctor del Doctorado en Humanidades, humanismo y persona, de la Universidad de San Buenaventura, Bogotá. Correo electrónico dariohuertas@gmail.com.
}

educación secundaria no valoran positivamente las clases que recibieron sobre Filosofía; es normal escuchar cómo esta materia les pareció "aburrida e intrascendente", con temas destinados a la formalidad del examen del ICFES, o con tópicos que no pasaron de ser curiosidades o temáticas pertinentes a otras clases como religión y orientación vocacional.

La opinión de docentes y estudiantes de Filosofía no dista mucho de lo anterior. Los profesores se quejan de la apatía de los alumnos. Siempre se percibe una carencia de los estudiantes, relacionada con su contexto formativo; así mismo, los estudiantes observan que muchos maestros tan solo cumplen con una función, distinta a la imagen ofrecida por la Filosofía de oportunidad de autorreflexión y examen de la realidad. Sin embargo, sostener opiniones de este tipo es un recurso fácil cuando no hay información de primera mano en relación al pensamiento y las prácticas de maestros, "la

Ruiz, Joaquín Darío Huertas. Enseñanza de la filosofía: ¿qué se le escapa al enfoque académico? Revista Sul-Americana de Filosofia e Educação. Número 30: nov./2018-abr.2019, p. 38-48. DOI: https://doi.org/10.26512/resafe.vizo.28238 
normatividad", la recepción de los estudiantes, en fin, la presencia de la Filosofía en el bachillerato.

La enseñanza de la filosofía es una problemática de gran actualidad en el campo profesional de la filosofía pues se discute su pertinencia en la educación: mientras algunos afirman la necesidad de su enseñanza a partir de la más tierna infancia, con el fin de erigir sujetos capaces de vivir con criterio en la sociedad democrática, hasta quienes niegan la necesidad de enseñarla, porque como disciplina profesional, requiere un entrenamiento destinado para unos pocos capaces de dominar la historia y las diversas temáticas de la filosofía. Entre estos dos extremos, encontramos tanto a los filósofos que enseñan y hacen investigación en las facultades e institutos de filosofía de las diversas universidades del país, como a los profesores de filosofía, quienes enseñan en las instituciones de enseñanza media, sosteniendo cualquiera de las posiciones determinadas, pero así mismo, proponiendo y desarrollando cursos de filosofía, tanto en la educación secundaria como en algunos cursos universitarios relacionados con filosofía.

Para el sistema educativo colombiano, la Filosofía hace parte del cuerpo de disciplinas obligatorias de la denominada Educación Media, junto con la Física y la Química, el Cálculo Matemático y la Literatura, haciendo parte de los conocimientos que permiten profundizar en el estudio de la naturaleza, para preparar al ejercicio de la ciudadanía, al mundo del trabajo y a los estudios superiores De acuerdo a la Ley General de Educación, la enseñanza de la Filosofía hace parte de los estudios obligatorios de la educación media (Cfr. MEN, 1983, Articulo 31). Su propósito coincide con los siguientes objetivos específicos: “a) La profundización en un campo del conocimiento o en una actividad específica de acuerdo con los intereses y capacidades del educando;

c) La incorporación de la investigación al proceso cognoscitivo, tanto de laboratorio como de la realidad nacional, en sus aspectos natural, económico, político y social;

f) El fomento de la conciencia y la participación responsables del educando en acciones cívicas y de servicio social;

g) La capacidad reflexiva y crítica sobre los múltiples aspectos de la realidad y la comprensión de los valores éticos, morales, religiosos y de convivencia en sociedad," (MEN, 1983).

La legislación, por lo tanto, parecería zanjar la discusión en favor de la relación educación y filosofía; sin embargo, la posición que adoptan los profesores de filosofía y quienes hacen investigación en filosofía, comporta una serie de problemáticas que merecen atención: ¿Qué es enseñar Filosofía? ¿Qué debe enseñarse en esta clase? ¿Se enseña realmente filosofía en la escuela? ¿Cómo concibe el profesor la filosofía, su enseñanza, los objetivos de esta?

El Ministerio de Educación, mediante el documento "Orientaciones pedagógicas para la enseñanza y el aprendizaje de la Filosofía" (MEN, 2009) (un documento preparatorio para la producción de lineamientos y estándares para la enseñanza de la Filosofía), asume como paradigmas de formación los modelos de la paideia (educación) griega y del bildung (formación) alemán; se constituye así una estructura curricular y didáctica desde un enfoque problémico, haciendo de la pregunta filosófica que pretende "filosofar y no enseñar Filosofía”, el eje fundamental; incorpora la interrogación a las didácticas habituales: exposición, disertación, 
diálogo socrático, análisis de textos, entre otros. El documento también propende por la perspectiva pedagógica y curricular de las competencias critica, dialógica y creativa, las cuales coinciden con las competencias argumentativas, comunicativas y propositivas, propias de las perspectivas pedagógicas actuales.

En general, la propuesta pide al maestro asumir la Filosofía como ámbito de reflexión a través del tiempo y las muy distintas maneras como se puede llevar la clase. Al ser una propuesta desde las competencias, se encuentra contradicción entre enseñar a filosofar y la idea de enseñar contenidos de la Filosofía, pues el documento finalmente privilegia el desarrollo de las competencias, de carácter instrumental, mediante múltiples estrategias didácticas que propenden por una perspectiva histórica, centrándose entonces en los contenidos producidos por los pensadores a través del tiempo como posibilidad de producir sujetos competentes. Es de preguntarse si es posible formar filosóficamente siguiendo los contenidos y las didácticas empleadas habitualmente. La propuesta del MEN olvida el contexto en el cual los estudiantes se encuentran y pretende seguir con una perspectiva creada en el siglo XIX.

Para los académicos, el papel de la Filosofía en la educación es necesario y urgente. Para Alejandro Cerletti, en su libro "La enseñanza de la Filosofía como problema filosófico" (CERLETTI, 20o8), la enseñanza de la Filosofía es en sí misma un problema que incumbe en su resolución no solo al maestro y la escuela, sino también a la Filosofía misma como reflexión del filósofo que enseña y al estudiante como destinatario de la formación filosófica y potencial pensador de su propia realidad. Cerletti apuesta por rediseñar el modo en que se aborda y se enseña la disciplina: se invita al maestro a crear nuevas didácticas y acceder a diversas propuestas filosóficas con el fin de ofrecer perspectivas de enseñanza y aprendizaje, donde el estudiante pueda pensar y actuar filosóficamente.

El autor pone de manifiesto las implicaciones pedagógicas, sociales y políticas de la Filosofía como educación: cómo la educación filosófica implica un maestro filósofo. La Filosofía está involucrada con un estilo de trabajo con los objetos que trata, los cuales son vastos como disciplina de estudio y como trato con la existencia del hombre; el filósofo aborda los problemas que le atañen con metodologías y preguntas de trabajo eminentemente filosóficas y ello hace que los problemas tomen perspectivas distintas; es evidente que el maestro de Filosofía no puede tratar un tema desde otra perspectiva que no sea filosófica, a pesar que en muchos medios como el nuestro esta clase es impartida por profesionales de otras disciplinas. Así mismo, el autor se cuestiona sobre el alcance de una educación realmente filosófica, en una sociedad donde sea posible un equilibrio entre lo establecido y el cambio y un Estado que permita pensar y actuar de manera creativa a sus ciudadanos. La situación de Sócrates en su tiempo, condenado y ejecutado por delitos al Estado unido al hecho de convertido en una especie de santo para el pensamiento, se ha visto como una paradoja entre las posibilidades de pensar libremente y lo que se puede pensar alrededor de tal libertad (CERLETTI, 2008).

Rafael Gómez Pardo, en su libro " $L a$ enseñanza de la Filosofía” (GÓMEZ, 2007), la propone como una problemática especialmente pedagógico ética. La Filosofía es entonces una formación para la vida buena, que debe tocar la existencia de los estudian- 
tes. Para Gómez, la enseñanza de la Filosofía debe tener clara su orientación formativa integral y esta debe favorecer tanto al individuo como a la sociedad. Su propuesta deriva hacia el desarrollo de didácticas en las que se privilegie el desarrollo del pensamiento filosófico, a partir de la vivencia cotidiana, para formar una actitud filosófica. La reflexión de este autor nos lleva a valorar el proceso de enseñanza desde el desarrollo de la capacidad filosófica y a estrategias que privilegien el diálogo y el examen racional de los intereses del estudiante.

La propuesta de la academia recae en un maestro en condiciones de formar y filosofar, así como en el estudiante ávido de formación, ambos en apertura crítica y transformadora de la realidad, trascendiendo los conocimientos habitualmente establecidos, convirtiendo la enseñanza de la Filosofía en una oportunidad de formación, en actitud reflexiva y filosófica autentica, tanto del docente como del estudiante.

Lo que se ha ponderado hasta ahora es, entre tantas otras propuestas, la idea establecida sobre el papel de la Filosofía en el aula: se espera que la Filosofía sea la panacea en el desarrollo del sujeto, le ayude a transformar su realidad y a adquirir pensamiento crítico y racional. Se asume que el estudiante es un recipiente vacío presto a la construcción de criterios e interesado por los contenidos de la disciplina. En el mismo sentido, se asumen como útiles y eficaces los contenidos propuestos para la enseñanza media y en general para la educación de los jóvenes ciudadanos. Al cruzar las opiniones de la gente del común con las propuestas del Estado y la academia, el resultado es contradictorio: quienes recibieron clase de filosofía en la educación secundaria e incluso en la educación superior en clases de ética o epistemología, por ejemplo, no estiman el resultado de esta enseñanza positivamente, podemos asumir rápidamente que los objetivos no se cumplen, ya sea responsabilidad de los maestros, de la enseñanza en general o de los estudiantes. En apariencia, no se logra el cometido con independencia del responsable de tal situación. Varias preguntas nos surgen desde esta perspectiva: ¿La Filosofía realmente está de más en el horizonte de la educación secundaria? ¿El maestro está al tanto y comprometido con las metas que le imponen los ideales o sencillamente no tiene una clara orientación de su trabajo, con todas las consecuencias que esto puede tener? ¿Los maestros conocen realmente la Filosofía como quehacer, no solo como saber, esto es, tener una verdadera actitud filosófica?

Frente a estos interrogantes se hace necesario acceder a la práctica cotidiana de los docentes, lo que origina el proyecto de investigación "La Enseñanza de la Filosofía como problema educativo" en el cual se estableció como objetivo general:

"Identificar y caracterizar las concepciones acerca la enseñanza de la filosofía y del papel del profesor de filosofía desde la perspectiva disciplinar y desde la propuesta del conocimiento profesional docente y sus implicaciones en las concepciones sobre la enseñanza y la formación de profesionales en filosofía."

A partir de este objetivo, se esperaba poder establecer las concepciones teóricas que fundamentan el ejercicio de la enseñanza de la filosofía en la educación media; revisar el estado del arte de la investigación en enseñanza de la filosofía, en el ámbito local y continental. Así mismo, se esperaba establecer los rasgos distintivos del pensa-

\footnotetext{
${ }^{2}$ Proyecto 007-005, "Enseñanza de la filosofía como problema educativo". Facultad de Filosofía. Universidad de San Buenaventura.
} 
miento profesional docente en la enseñanza de la filosofía.

Los resultados de este ejercicio son de gran interés debido a que manifiestan la necesidad de revisar las concepciones y enfoques que se poseen sobre la enseñanza de la filosofía, tanto los maestros en ejercicio como las instituciones que forman a los futuros cuadros docentes. También es relevante resaltar cómo las experiencias de los profesores de filosofía en las aulas son prácticamente desconocidas y desaprovechadas por la academia, cuestión que implica la necesidad de explorar la práctica docente en las instituciones educativas.

\section{La propuesta metodológica.}

El proceso de acopio de la información y el establecimiento de la parte teórica, se planteó desde la revisión de documentos específicos acerca de la enseñanza de la filosofía y manuales de didáctica de la filosofía, esperando que las fuentes escogidas ofrecieran un horizonte de comprensión, pero ante todo una guía medianamente clara acerca del modo en que se concibe en Colombia enseñar filosofía. Se esperaba que el trabajo de recolección de datos no encontrara mayores obstáculos que el volumen de la información disponible contra el tiempo de desarrollo del proyecto de investigación.

El levantamiento de las concepciones teóricas sobre la enseñanza de la filosofía se planeó como una indagación de carácter bibliográfico de monografías, sistematizaciones, tesis de maestría y de doctorado, comenzando por las bases de datos y catálogos de las universidades donde existen programas de filosofía, siguiendo con la confrontación de los trabajos depositados en las bibliotecas y seleccionando luego algunos de estos trabajos como representativos del estado de la cuestión en la forma- ción para la enseñanza de la filosofía. Así mismo, se buscaron las publicaciones especializadas relacionadas con la enseñanza de la filosofía, con la pretensión de encontrar una abundante documentación del tema.

\section{El resultado de la indagación.}

A partir de la relación de la bibliografía seleccionada para el marco teórico y de la referencia a los trabajos de grado y la literatura vigente, se esperaba poder establecer una perspectiva clara acerca de la enseñanza de la filosofía en la educación media en Bogotá, las experiencias realizadas en este aspecto en las prácticas y colegios de la ciudad, los resultado de la investigación, etc. Todo ello con el fin de proporcionar un estado de la cuestión susceptible de entrar en conversación con el programa de investigación denominado pensamiento profesional docente, el cual ofrece una perspectiva enriquecedora para la formación profesional de nuevos cuadros profesionales.

Se esperaba que, por lo menos en Bogotá, que cuenta con alrededor de 16 programas de licenciatura en filosofía o programas de formación profesional en filosofía, con 6 programas de maestría en filosofía y tres programas de doctorado en filosofía, la búsqueda ofreciera un volumen apreciable de tales publicaciones. Sin embargo, una pesquisa amplia en las bibliotecas y bases de datos arrojaron una docena (12) de trabajos de grado que trataron aspectos de la didáctica de la filosofía, o experiencias de enseñanza de la filosofía; se encontraron quince tesis de maestría y ninguna de doctorado. Esta primera indagación fue reconfirmada por otros dos intentos de búsqueda que arrojaron resultados similares, incluso ampliando el margen de búsqueda dos décadas atrás. Si bien faltó por confirmar la existencia de algunos volúmenes por la dificultad para acceder a ellos en 
algunas bibliotecas de las universidades, consideramos que un número tan pequeño de trabajos de grado y tesis de maestría, en una comunidad profesional tan amplia, trabajando como maestros, puede manifestar algunas cuestiones interesantes.

Aparte de los trabajos de grado, la búsqueda de publicaciones arrojó un resultado similar, teniéndose en cuenta que aparecen títulos publicados en el país como en otras naciones, especialmente de la Argentina, México y España, mientras que la publicación de libros relacionados con el tema en Colombia se han centrado en las universidades de San Buenaventura con cuatro títulos, Pedagógica Nacional, con una serie de varios tomos de recopilaciones de artículos de la línea "Filosofía y enseñanza de la filosofía” de su doctorado en educación y la Universidad de Antioquia, con dos títulos específicos sobre la didáctica de la filosofía. La publicación de artículos en revistas especializadas en Filosofía es creciente y versa sobre todo en reflexiones generalizantes acerca de la enseñanza de la filosofía, sin incluir, salvo contadas excepciones, temas referentes a las técnicas didácticas para la enseñanza de la filosofía. Sin embargo, comparando con el volumen de publicaciones existentes sobre temas filosóficos en el país, sorprende por su escasez a lo largo del tiempo.

\section{El análisis.}

Frente a este preocupante panorama, donde el desarrollo del conocimiento de la didáctica y la enseñanza de la filosofía es precario, los interrogantes no van a cuestionar tal escasez, sino a comprender las razones por las cuales se presenta tal ausencia. Ello originó la necesidad de replantear objeto de la investigación, en tanto la ausencia de investigación sugiere que la temática ha carecido entonces de significación para el gremio de filósofos y profesores de filosofía, en términos de proponer el problema de la enseñanza de la filosofía, que enseñan esta disciplina en las instituciones de educación media de nuestro país, e incluso en el continente salvo algunas excepciones significativas como los trabajos de Paredes en la Universidad de Antioquia, Cerletti, Kohan y Obiols, en el Cono sur.

A medida que se iba haciendo lectura de los textos emergen dos enfoques en la concepción de la enseñanza de la filosofía, tanto en su estructura teórica como en el modo en que se sugiere enseñar en el salón de clases, a saber: un enfoque teorizante, que emerge de las facultades de Filosofía, en las condiciones de investigación y producción de la universidad, y otro enfoque, producto de la experiencia en el aula, bajo las condiciones y pautas que tiene la educación como institución y que provea de un fundamento filosófico a los estudiantes. En otras palabras, un enfoque académico y uno práctico.

El análisis deja como punto de partida, la necesidad de caracterización de ambos enfoques, para precisar así sus diferencias y definir lo que se escapa a la posición académica; tal enfoque incluye tanto los objetivos de la Filosofía en la educación, como algunos temas tratados por la academia, referidos tanto a la función de la Filosofía en la escuela, como a las didácticas, contenidos a abordar en el proceso educativo, entre otras cosas.

La hipótesis emergente es que la concepción de la enseñanza de la Filosofía desde la academia, no está orientada por la investigación y la reflexión sobre los actos e intereses de los actores que intervienen en ese "pequeño universo" que es el aula; por ello se vuelve un tema tratado desde un en- 
foque romántico que no ha tenido en cuenta la experiencia en el aula y no ha ofrecido directrices que sean provechosas para hacer de la Filosofía propiamente un saber rector y transformador de la educación y las actitudes de los individuos en general.

\section{El enfoque académico.}

La producción acerca de la enseñanza de la filosofía en nuestro medio, es esencialmente teórica. Lo escrito procede de lo producido por la investigación filosófica, pero tal investigación está encaminada hacia la reflexión, que dejan de lado elementos de la cotidianidad necesarios para la comprensión del acontecimiento que es enseñar filosofía. Ello produce que las reflexiones, si bien están encaminadas hacia la filosofía, razonan el quehacer filosófico y el dominio del filosofar, pero desconoce las necesidades de quienes reciben la formación filosófica en la educación secundaria, sus contextos, las habilidades necesarias para la vida que desarrollaría el trato con la filosofía. Podemos citar algunos ejemplos de tal controversia, no sólo en nuestro país, sino también en el ámbito internacional: Rafael Gómez Pardo, en su libro La enseñanza de la Filosofía (2007), analiza el acto filosófico de la educación y cómo este se convierte en un problema importante en la formación de los individuos y la sociedad como una formación para la vida. Analiza cómo la enseñanza de la Filosofía se convirtió en la enseñanza de historia de la Filosofía, divorciando al saber del ser, distinguiendo la labor filosófica de la pedagógica, que Pardo considera una sola. En el mismo orden de ideas, Miguel Ángel Gómez, en su libro Introducción a la Didáctica de la Filosofía (2003), se pregunta acerca de la posibilidad de una didáctica de la filosofía, en relación a la diferencia existente entre la filosofía y la didáctica, entre los fines del quehacer filosófico y las características del saber práctico que posee la didáctica, proponiendo la prevalencia de una didáctica que surja del trabajo propio de la investigación filosófica, conduciendo de este modo al ejercicio filosófico educativo. Concepciones similares encontramos en German Vargas, quien ha publicado varios libros acerca de la relación entre filosofía y educación, validando la necesidad de la filosofía en la escuela y afirmando su carácter formativo, en el desarrollo de la ciudadanía, el sentido ético, lo definidamente humano, etc.

La concepción del profesor Domingo Araya, en su texto Didáctica de la historia de la filosofía (2004), comprende la filosofía como un saber que se aprende al tomar conciencia de su historicidad, la cual puede ser aprendida por etapas históricas y en el conocimiento de sus personalidades, efemérides y anécdotas, planteamiento que comparten numerosos profesores de filosofía. Ello implica que habrá gran diferencia entre quien hace la filosofía y quien aprende la filosofía.

En este sentido, las concepciones propuestas desde la academia, se generan desde la idea de una producción filosófica que contempla su enseñanza como una producción reflexiva, esto es, un ejercicio natural de investigación que comprometería una posición definida del estudiante ante su realidad. Las opiniones van desde la capacidad de la Filosofía de generar tales condiciones en el individuo de manera automática, prácticamente a partir de la lectura de las grandes obras o del examen de grandes autores, hasta la aplicación de las metodologías propias de las escuelas filosóficas. Sin embargo, es necesario reconocer de un modo pragmático, que tal forma de enseñanza, si no es utópica, por lo menos es poco implementable en el contexto educativo en el cual estamos: el número de estudiantes es 
siempre elevado, los tiempos de clase exiguos, los materiales para la clase, precarios.

En lo que se equivoca la academia es en pensar que la educación básica y media es el mismo escenario que la educación superior y la investigación filosófica. Las necesidades de la educación, sobre todo con las políticas y estrategias del estado en cuestión de resultados y procesos de medición, nos ponen frente a una orientación equivocada de la enseñanza de la Filosofía. Aquí está precisamente lo que se le escapa a las consideraciones de la academia: los contextos escolares de enseñanza y aprendizaje, no se consideran como problemas de investigación filosófica ni educativa, sin considerar las diferencias entre las condiciones específicas de la filosofía profesional no son inaplicables a la práctica de la filosofía con los estudiantes de la educación básica y media, quienes no tienen las herramientas ni las habilidades para filosofar como se espera de quienes tratan con la disciplina.

La propia experiencia en la educación media sugiere que los profesores de filosofía desarrollan prácticas diversas a las propuestas por la literatura académica. Los profesores de filosofía chocan con la realidad que implica filosofar con los estudiantes, cuestión que no está documentada en la literatura sobre la enseñanza de la filosofía y que se constatan en los resultados que generan en las pruebas estatales, que miden la calidad de la educación.

\section{Los contextos escolares. ¿La experiencia en el aula, reclamos sin eco?}

Las clases de Filosofía y los maestros de Filosofía son criticados por no ofrecer mayores condiciones para la formación del individuo ni para su participación en la sociedad. La enseñanza de la Filosofía entonces no pasa de ser un contenido que no ofrece mayor beneficio en la educación de las juventudes que se forman en la educación básica. Es por esto que nos preguntamos: ¿El contacto con la Filosofía no genera una actitud de conversión de la existencia, evocando a Platón, de salida de la caverna? ¿Qué suscita en los estudiantes el contacto con la Filosofía y cómo esta trasciende las clases del bachillerato en las vidas cotidianas de las personas?

En un trabajo anterior presenté algunas impresiones de maestros y estudiantes sobre lo que significaba para ellos la clase de Filosofía; encontramos que si bien la Filosofía despertaba cierta atracción al público joven, esta desaparecía tan pronto se iniciaban las clases; los estudiantes miraban con alguna simpatía a sus maestros de Filosofía, pero no encontraban en ellos mayor provecho. De parte de los profesores, estos no encontraban eco en sus esfuerzos y estimaban que la recepción de su enseñanza no satisfacía en ningún momento lo que ellos esperaban. Para este tópico, la Filosofía sólo es un contenido más, sin mayor trascendencia, el cual no propone mayor cosa al estudiantado, mientras los maestros se esfuerzan por enseñar un saber para el que no tienen las herramientas que les permitan responder a los requerimientos del estado y a los de los individuos.

El contexto escolar en que nos encontramos en la escuela condiciona la enseñanza no sólo de la Filosofía sino de todas las clases: ambientes violentos, junto a la confusión reinante en lo relacionado a la formación, los derechos, la convivencia, son contextos propicios y hostiles a la reflexión filosófica. Los movimientos sociales, los medios y los nuevos ambientes virtuales y de la comunicación, son ambientes inexplorados por la filosofía para ser usados como medio y mediación en la enseñanza y el 
aprendizaje de una concepción crítica de la realidad. Así mismo, el control moral de los medios, es mencionado y denunciado por la filosofía como un anti humanismo, pero no se usa para que los estudiantes de la secundaria se ejerciten en el filosofar. El contexto obliga entonces a transformar la concepción ortodoxa de la filosofía para hacer una crítica que transporte el contexto.

\section{Las condiciones y experiencia de los maestros.}

La literatura sobre sistematización de experiencias de los profesores de filosofía, es casi inexistente, como ya se dijo, existen trabajos de grado que abordan alguna estrategia didáctica, pero sin hacer un proceso de reflexión y conceptualización que ofrezca aportes a la didáctica. Los artículos que sistematizan las experiencias de los maestros de filosofía, son prácticamente inexistentes, salvo el significativo trabajo hecho por el grupo de investigación de la Universidad de Antioquia, hace unos seis años.

Pero eso no quiere decir en ningún momento, que los maestros estén despreocupados por su quehacer. Conversaciones informales con docentes, manifiestan una gran preocupación por la formación filosófica de los estudiantes, creando alternativas didácticas para enseñar efectivamente 3 , las cuales lastimosamente se pierden porque no se ha hecho una labor de sistematización de tales experiencias, en parte debido a la perspectiva academicista que no ha considerado la investigación en el aula como parte del desarrollo de la enseñanza de la filosofía.

\footnotetext{
3 Se ha propuesto y aprobado para adelantar el proyecto de investigación "El pensamiento profesional de los profesores de Filosofía en la localidad de Usaquén”, en la Universidad de San Buenaventura.
}

Por ello, es necesario proponer trabajos de investigación que recobren este saber metodológico didáctico y permitan una transformación del quehacer educativo de la filosofía. Del mismo modo, es importante conocer e interpretar cuáles son las concepciones que tiene el maestro acerca de su labor; hemos creído que en la formación ofrecida por los programas y currículos de las licenciaturas están claras las orientaciones y el sentido de enseñar filosofía en la escuela, así como los insumos pedagógicos para optimizar su labor educativa, pero tanto la experiencia propia como el diseño de las pruebas de estado, demuestran que existen vacíos importantes en esos aspectos: podemos tener en cuenta el reducido número de estrategias didácticas específicamente filosóficas, y el desconocimiento de los distintos modelos evaluativos y su relación con las didácticas y las pedagogías. Vale como cuestionamiento lo siguiente: ¿de qué modo asignamos una valoración evaluativa a los estudiantes? ¿Bajo qué parámetros? Metodológicamente ¿cómo procedemos para conciliar información, actividades con la pretensión del quehacer filosófico?

\section{Conclusiones}

La academia ha pensado bastante la relación entre la educación y la Filosofía, pero le hace falta hacer una reflexión a fondo sobre lo que implica la Filosofía como parte del proceso educativo. La enseñanza de la Filosofía es uno de los campos de acción de la Filosofía, de hecho, puede ser el campo que ocupa a más profesionales de este saber: en el caso específico de nuestro país, de las varias decenas de programas de formación activos en el país, la mayoría de sus egresados ejercen en instituciones educativas de secundaria y otro tanto en la formación humanística, ética, etc. En la formación de la educación superior. Lo 
bueno de esta situación, por lo menos en nuestro país, es que hay un amplio espectro de trabajo para los egresados.

Frente a los temores que subyacen a un enfoque académico de la enseñanza de la filosofía como, por ejemplo, el quehacer especifico del filósofo, cabe decir que este tipo de producción de conocimiento nos acerca del mismo modo a la forma en que producimos un saber de carácter práctico, el cual no se valida en un ensayo o un libro, sino en el uso de la filosofía en la vida cotidiana de un joven. Como muestra de la importancia de este punto, mencionemos que los diálogos y las historias más edificantes de algunos filósofos de la antigüedad, son precisamente recopilaciones de las experiencias vividas por los primeros filósofos reconocidos, como los recogidos por Diógenes Laercio y los reconocidos diálogos platónicos, protagonizados por Sócrates.

\section{Referencias}

AGUILAR, L. A. Conversar para aprender. Gadamer y la educación. En: Revista Electrónica Sinéctica, (23), 2004, p. 11-18. Disponible en: http://redalyc.uaemex.mx/src/inicio/ArtPdfRed.jsp?iCve=99815908003

ARAYA, D. Didáctica de la historia de la filosofía. Bogotá: Magisterio, 2004.

BEYER, L. William Heard Kilpatrick (1871-1965). Perspectivas: Revista trimestral de educación comparada. UNESCO. XXVII (3), 1997, p. 503-521.

CERLETTI, A. La enseñanza de la Filosofía como problema filosófico. Buenos Aires: Los libros del zorzal, 2008.

CHEVALLARD, Y. La transposición didáctica. Del saber sabio al saber enseñado. Buenos Aires: Aiqué, 1997.

DUSSEL, I.; PINEAU, P.; CARUSO, M. La Escuela como máquina de educar: tres escritos sobre un proyecto de la modernidad. Buenos Aires. Paidós, 2001.

FREIRE, P. Educación como práctica de la libertad. Santiago, 1965.

GADAMER, H. G. Sobre los que enseñan y los que aprenden. En: La herencia de Europa. Barcelona: Península, 2000, p. 145-150.

GADAMER, H.-G. Verdad y Método. Fundamentos de Hermenéutica filosófica. Salamanca: Sígueme, 1988.

GÓMEZ, M. Á. Introducción a la Didáctica de la Filosofía. Pereira: Papiro, 2003.

GÓMEZ, R. La enseñanza de la Filosofía. Bogotá: Universidad San Buenaventura, 2007.

KILPATRICK, W. H. La función social, cultural y docente de la escuela. Buenos Aires: Losada, 1946.

GÓMEZ, A. L. ¿Enseñar Filosofía? Santiago de Cali: Universidad del Valle, 2008. 
MARCELO, G. C. La investigación sobre el conocimiento de los profesores y el proceso de aprender a enseñar. Una revisión personal. En: PERAFÁN G. A.; ADURÍZ-BRAVO, A. (ed.). Pensamiento y conocimiento de los profesores. Debate y perspectivas internacionales. Bogotá: UPN-Colciencias, 2002.

MEN. Ley general de Educación, 1983.

OBIOLS, G. Una introducción a la enseñanza de la filosofía. Buenos Aires: Los libros del zorzal, 2008.

ONFRAY, M. La comunidad filosófica. Barcelona: Gedisa, 2008.

. Antimanual de Filosofia. Madrid: EDAF, 2005.

ORTEGA, J. Memoria, hermenéutica y educación. Madrid: Bilbioteca Nueva, 2002.

PAREDES, D. M., \& RESTREPO, V. V. Enseñanza de la filosofía en Colombia: hacia un enfoque multisensorial en el campo didáctico. Nodos y nudos. v, 4 (34), 2013, p. 37-48.

PERAFÁN, G. A. La epistemología del profesor sobre su propio conocimiento profesional. Bogotá: Universidad Pedagógica Nacional, 2004.

PORLÂN, R., \& RIVERO, A. El conocimiento de los profesores. Sevilla: Diada Editora, 1998.

UNESCO. Recomendaciones en el ámbito de la enseñanza de la filosofía en América Latina y el Caribe. Manifiesto de la Reunión de Alto Nivel sobre la Enseñanza de la Filosofía en América Latina y el Caribe, 2009.

SALAZAR BONDY, A. Didáctica de la filosofía. Lima: UNMSM, Facultad de Letras y Ciencias Humanas, Fondo Editorial, 1995.

SHULMAN, L. S. Knowledge and teaching: foundations of new reform. Harvard, 1987.

SHULMAN, L. S. Paradigmas y programas de investigación en el estudio de la enseñanza. Una perspectiva contemporánea. En: WITTROCK, M. C. La investigación de la enseñanza

I. Barcelona: Paidós, 1989, p. 9-91.

VALBUENA U. E. El conocimiento didáctico del contenido biológico: estudio de las concepciones disciplinares y didácticas de futuros docentes de la Universidad Pedagógica Nacional. (Colombia). Madrid: Universidad Complutense de Madrid. 2007. Tesis doctoral

VARGAS, G. Espacios de acción de la filosofía en la sociedad. En: ¡Enseñar Filosofía! Bogotá: UPN, 2007, p. 9-23.

UPN, 2004.

; CÁRDENAS, L. (Ed.) Filosofía, pedagogía y enseñanza de la filosofía. Bogotá.

VILLAR, L. M. Pensamientos de los profesores Pensamiento y Conocimiento de los profesores. Bogotá: UPN COLCIENCIAS, 2002, p. 29-44. 